DOI:10.2478/rrlm-2020-0015

\title{
Implications of visfatin genetic variants in the metabolic profile of the Romanian pediatric population
}

\author{
Simona Loredana Vasilache ${ }^{1}$, Cristina Oana Mărginean ${ }^{2}$, Anastasia Boaghi ${ }^{3}$, \\ Raluca-Monica Pop ${ }^{4}$, Claudia Banescu ${ }^{5,6^{*}}$, Valeriu G Moldovan 6 , Adina Hutanu6, \\ Carmen Duicu ${ }^{2}$, Ionela Maria Pascanu ${ }^{7}$ \\ 1. George Emil Palade University of Medicine, Pharmacy, Science, and Technology \\ of Targu Mures, Romania \\ 2. Department of Pediatrics, George Emil Palade University of Medicine, Pharmacy, Science, \\ and Technology of Targu Mures, Romania \\ 3. Department of Endocrinology, Mures County Hospital Targu Mures, Romania \\ 4. Department of Endocrinology, Mures County Hospital Targu Mures, Romania; Department of \\ Research Methodology, George Emil Palade University of Medicine, Pharmacy, Science, \\ and Technology of Targu Mures, Romania \\ 5. Department of Genetics, George Emil Palade University of Medicine, Pharmacy, Science, \\ and Technology of Targu Mures, Romania \\ 6. Genetics Laboratory, Center for Advanced Medical and Pharmaceutical Research, George Emil \\ Palade University of Medicine, Pharmacy, Science, and Technology of Targu Mures, Romania \\ 7. Department of Endocrinology, Mures County Hospital Targu Mures, Romania, Department of \\ Endocrinology, George Emil Palade University of Medicine, Pharmacy, Science, and Technology of \\ Targu Mures, Romania
}

\begin{abstract}
Background: Conflictual results regarding the relationship between plasmatic level of visfatin and obesity could be explained by the influence of the gene variants involved in the synthesis or action of these hormones. Objectives: The present study examined the potential implication of single nucleotide polymorphisms (SNPS) of nicotinamide phosphoribosyltransferase (NAMPT) gene that encodes visfatin, in obesity, in a Romanian pediatric population. Method: A case-control study was conducted on a group of 213 children, divided into two: the case group - 130 overweight and obese children with BMI $>1 S D$, and the control group - 83 children with normal BMI. The variables analyzed were age, sex, anthropometric parameters, body composition based on bioimpedance analysis, lipid profile, visfatin and insulin plasmatic levels, rs4730153 and rs2302559 visfatin SNPs. Results: Significant associations were not found between rs4730153 and rs2302559 visfatin SNPS and obesity. Regarding lipid metabolism, there are statistically significant differences between triglyceride levels according to NAMPT rs2302559 genotypes
\end{abstract}

*Corresponding author: Claudia Banescu, Department of Genetics, George Emil Palade University of Medicine, Pharmacy, Science, and Technology.of Targu Mures, Romania. E-mail: claudia.banescu@gmail.com 
$(p=0.045)$, with heterozygous genotype having the highest level of triglycerides, and also between cholesterol levels according to NAMPT rs4730153 genotypes ( $p=0.030$ ), with carriers of heterozygote genotype having the highest level of cholesterol. There is a statistically significant difference between the studied parameters in the investigated groups, regarding cholesterol, in carrier of wild-type genotype of NAMPT rs2302559 ( $p=0.040)$ and carrier of wild-type genotype of NAMPT rs4730153 ( $p=0.036$ ). We observed no association of NAMPT rs 4730153 and rs2302559 with visfatin levels in the studied groups. Visfatin level was lower in the case group and was correlated with weight $(p=0.042)$, abdominal circumference $(p=0.010)$, waist to height ratio $(p=0.040)$, but not with the elements of the metabolic profile. Conclusion: NAMPT rs2302559 and rs4730153 polymorphisms do not seem to have a major impact in the development of obesity in children, however there may be an association with lipid profile, but further studies are needed..

Keywords: visfatin, insulin, visfatin polymorphisms, pediatric obesity

Received: $7^{\text {th }}$ September 2019; Accepted: $3^{\text {rd }}$ February 2020; Published: $7^{\text {th }}$ February 2020

\section{Introduction}

Obesity in children, adolescents, and adults is considered to be the most important public health problem in recent years $(1,2)$. The World Health Organization (WHO) estimates that in 2025 approximately 70 million children will be overweight or obese unless adequate prevention and control measures are applied. Romania occupies the third place in Europe in terms of obesity incidence according to IOTF (International Obesity Task Force) (3-5). The PREDATORR study showed an increase in the prevalence of obesity in the adult population in Romania. The study performed on 2681 patients, a cohort statistically representative for the entire adult population of Romania, revealed that the prevalence of obesity was $31.90 \%$ and the prevalence of abdominal obesity was $73.90 \%$ (6). If in $200915 \%$ of Romanian children were obese, in 2015 the percentage increased to $25 \%$. A pooled analysis of data on body mass index BMI disturbances in Romanian children from 2006 to 2015 revealed that the prevalence of children with obesity was $28.3 \%$, with the highest prevalence in children $6-12$ years of age $(34 \%)$ and the lowest in the 15-19 year-old group (17\%) (7).

Based on the latest research, the American Association of Clinical Endocrinologist (AACE) and the American College of Endocrinology (ACE) proposed a new terminology for obesity: adiposity-based chronic disease (ABCD). Many researchers have extensively studied the adipose tissue, however, the attention has been increasingly focused on the term "adiposity", which includes the function, distribution and the total quantity of adipose tissue (8).

Although it was known that adipose tissue secreted peptides, the number of adipokines has increased rapidly and includes adiponectin, resistin, visfatin, retinol binding protein 4 (RBP4), and chemerin. The secretion and preferential expression of pro-inflammatory molecules (visfatin, RBP4) was demonstrated in the adipose tissue of adult obese persons. All these adipokines have an important role in lipid homeostasis, blood pressure control, insulin sensitivity, coagulation, and have an essential contribution to obesity caused complications (9).

Insulin resistance (IR) appears to be an important disorder in the pediatric population. The pathway by which IR occurs is unclear, but studies have categorically argued that patients with IR are susceptible to metabolic syndrome development, type 2 diabetes and cardiovascular disease (10). The homeostasis model assessment for insulin resistance (HOMA-IR) index 
is generally used for defining IR in the adult population and has been applied in the pediatric population as well (11).

Visfatin was discovered as a growth factor for B lymphocyte precursors. It is encoded by the nicotinamide phosphoribosyltransferase (NAMPT) gene located on the long arm of chromosome 7. It is an adipokine secreted by the visceral adipose tissue, but also by macrophages within the adipose tissue, hepatocytes, as well as skeletal muscles (12-14), and it has been suggested that it might have both endocrine and paracrine effects. The hypothesis regarding the role of macrophages in the production of visfatin is supported by differences in visfatin production in the visceral and subcutaneous adipose tissue. It appears that RNA expression is identical in the visceral and subcutaneous adipose tissue in normal weight patients. Differences occur in obese patients, where visceral adipose tissue becomes a more potent visfatin producer, while it decreases at subcutaneous level (12). It was reported that visfatin acts on the insulin receptor by binding to another site than that of the usual substrate, but this hypothesis is still under debate (15).

In the pediatric population, the metabolic significance of visfatin remains unclear. The conflicting results regarding the relationship between insulin plasmatic levels as well as visfatin and obesity could be explained by the influence of the different gene variants involved in the synthesis or action of these hormones.

The latest studies have shown a genomic association of 227 gene variants involved in many biological pathways (appetite and digestion, adipocyte differentiation, insulin pathways, intestinal microbiota), and obesity (16). The link between some visfatin gene variants and obesity has been confirmed in several populations, but not in Romanian children (17). For the present study we selected rs4730153 and rs2302559 visfatin gene polymorphisms because there are studies in the adult population that have shown their involve- ment in the metabolic profile. However, studies in the pediatric population are extremely limited and the visfatin gene polymorphisms we have chosen have not been studied in the Romanian pediatric population. Thus, given the high prevalence of pediatric obesity in our country, the aim of the current study was to evaluate the associations between two single nucleotide polymorphisms (SNPs) for visfatin rs4730153 and rs2302559, and other metabolic abnormalities in overweight, obese, and normal weight children.

\section{Materials and Methods}

\section{Study design}

An observational study was conducted on 213 children, from three schools from Târgu Mures, Pediatrics Clinic no.1, and Endocrinology Clinic from Targu Mures, aged between 5 and 18 years, divided into two groups: the study group - 130 overweight and obese children with BMI $>1$ SD and the control group - 83 children with normal BMI, who were evaluated between 2016 and 2018. Inclusion criteria were: age between 5 and 18 years; study group: overweight (BMI $>+1 \mathrm{SD}$, according to the WHO) (18) or children with obesity (BMI $>+2$ SD); control group (BMI SD between -1 and +1 SD). Exclusion criteria were: history of severe chronic disease (cardiac disease, liver failure, renal insufficiency or malabsorption syndromes: celiac disease, short bowel syndrome, inflammatory bowel disease, as well as any known monogenic or chromosomal genetic diseases), children with short stature (height $\leq-2.5$ SD (based on the Prader, 1989 growth charts) (19), untreated endocrine disease, failure to obtain informed consent from the parents.

All parents signed a written consent for their children. The study was approved by the Ethics Committee of the University (No. 7/2016) and it was compliant with the principles of the Helsinki Declaration. 


\section{Method}

Upon written consent, all children were auxologically evaluated: height, weight, waist circumference, tricipital skinfold, using standardized tools and measurement protocols (20). The second step was the bioimpedance analysis after which blood was drawn for biochemical and genetic analysis.

The following variables were included: age, sex, anthropometric parameters: standard deviation score (SD) of body mass index (BMI), abdominal circumference (AC), tricipital skinfold thickness (TST), waist to height ratio (WHtR), body composition based on bioimpedance analysis (fat mass, free-fat mass), blood glucose, lipid profile (total cholesterol, HDL cholesterol, triglycerides), uric acid, visfatin and insulin plasmatic levels, HOMA index, 2 SNPs for visfatin namely, rs4730153 and rs2302559.

For waist circumference, the mean of two values was used. The position of the tape was approximately the midpoint of the line between the top of the iliac crest and the lower margin of the last palpable rib, (21). The available reference values were used (22) defining abdominal obesity as values above as previously described $+2 \mathrm{SD}$. The waist circumference/height ratio was used as a second definition for abdominal obesity with a cut-off value of 0.5 (22). Tricipital skinfold thickness was measured from the left arm, using a digital caliper, with a precision of 0.1 $\mathrm{mm}$, and was interpreted as normal $-1 \mathrm{SD}-+1$ $\mathrm{SD}$, overweight $+1 \mathrm{SD}-+2 \mathrm{SD}$ and obesity $\geq$ $+2 \mathrm{SD}(22)$.

Body composition based on bioimpedance analysis (using the Tanita BC-420 analyzer): the principle of this method is to measure the electrical resistance of the tissues of the body, determining the values corresponding to the quantity and percentage of each tissue type. Interpretation: for children under 16 years of age, obesity is defined as fat mass that exceeds the reference value for age and gender by more than $20 \%$; for children over 16 years the cut-off value for fat mass is $25 \%$ of the body weight in boys and $32 \%$ in girls (23).

Metabolic parameters analyzed: glucose levels; lipid profile: triglycerides, cholesterol, HDL cholesterol, uric acid. The biological tests for metabolic determinations were performed in the morning, after a fasting period of at least 8 hours. The reference range for all these metabolic parameters was established according to the cut-off values of the hospital.

Blood collection for biomarker assessment was performed in EDTA tubes. The samples were centrifuged for 15 mins at $1500 \mathrm{~g}$, aliquoted in safe-lock tubes and stored at $-80{ }^{\circ} \mathrm{C}$ until analysis. Visfatin plasma levels (Visfatin, Sigma-Aldrich, USA) and insulin (Human Insulin, DRG Instruments Germany) were measured on an automated DSX ELISA instrument (Dynex Technologies, USA) using the enzyme-linked immunosorbent assay (ELISA) according to the manufacturer's instructions and as previously described (24). The analytical sensitivity for Insulin was $1.76 \mu \mathrm{UI} / \mathrm{ml}$ and coefficients of variation $(\mathrm{CVs})$ for intra-assay and inter-assay precision were $<2.6 \%$ and $<3.0 \%$ respectively HOMA index (calculated with formula (glycemia $\mathrm{x}$ insulin)/405) (25), was interpreted as: $<2$ normal, $>2$ possible insulin resistance, $>2.5$ increased probability of insulin resistance (26). For visfatin, the minimum detectable concentration was $0.778 \mathrm{ng} / \mathrm{ml}$, while $\mathrm{CVs}$ were $<10 \%$ for intra-assay precision and $<15 \%$ for inter-assay precision.

Genotyping analysis: the Quick-DNA miniprep kit (ZymoResearch, USA) was used for processing fresh whole blood collected in EDTA from children in order to obtain the genomic DNA which was used for genotyping analysis. Two SNPs for visfatin within the NAMPT gene, namely rs4730153 (-3186C $>\mathrm{T})$ and rs2302559 $(-948 \mathrm{G}>\mathrm{T})$ were genotyped using specific TaqMan assays and an Applied Biosystems 7500 
Fast Dx Real-Time PCR instrument from ThermoFisher Scientific (USA).

Statistical analysis: for data collection, the Microsoft Office Excel software was used. The statistical analysis was performed using SPSS v. 25.0 (SPSS Inc, Chicago, IL, USA). For data display of continuous variables, the mean \pm standard deviation was used for normally distributed data, and median and interquartile range for non-normally distributed data. Categorical variables were expressed as frequencies and percentages. For mean comparison, the Mann-Whitney test was used; associations were tested using Fisher's exact test. Deviations from the Hardy-Weinberg equilibrium for genotype frequencies were tested using the chi-square test. Spearman coefficient was used for correlation analysis. For all analysis, statistical significance was obtained if $\mathrm{p}<0.05$.

\section{Results}

\section{Demographic characteristics}

The general characteristics and metabolic profile of the studied groups are described in Table 1, with no significant differences between median age and sex ratio in the two groups. Median age of the case group was 11.5 years (9.1-13.4) and 12.0 years for the control group (9.1-14), with a sex ratio Male:Female $=1.1$ for the case group and 0.8 for the control group $(\mathrm{p}=0.262)$.

There were significant differences between the two groups regarding the anthropometric parameters, all body composition parameters and metabolic parameters (cholesterol, triglyceride, HDL cholesterol, uric acid, insulin, HOMA index and visfatin), however, regarding glucose level there was no significant difference between the two groups $(\mathrm{p}=0.188)$ (Table 1$)$.

\section{Hormonal characteristics}

The HOMA index was significantly higher in the case group compared with the control group ( 7.58 vs $4.66, p<0.001)$. The HOMA index was significantly correlated with weight $\left(r_{\mathrm{s}}=0.355, \mathrm{p}=0.001\right)$, BMI $\left(\mathrm{r}_{\mathrm{s}}=0.390, \mathrm{p}=0.001\right)$, abdominal circumference $\left(\mathrm{r}_{\mathrm{s}}=0.386, \mathrm{p}=0.001\right)$, WHtR $\left(\mathrm{r}_{\mathrm{s}}=0.333, \mathrm{p}=0.001\right)$, skinfold $\left(\mathrm{r}_{\mathrm{s}}=0.230\right.$,

Table 1. Anthropometric and metabolic comparison between study groups

\begin{tabular}{lccc}
\hline & Case (BMI $>$ 1 SD) & Control (BMI $<$ 1 SD) & p value \\
\hline BMI SD & +2.48 & -0.10 & $<0.001$ \\
\hline Waist SD & +2.54 & +0.75 & $<0.001$ \\
\hline WHtR & 0.58 & 0.46 & $<0.001$ \\
\hline TST SD & +2.97 & -0.54 & $<0.001$ \\
\hline Fat-Mass $(\mathrm{kg})$ & 22.44 & 7.56 & $<0.001$ \\
\hline Free Fat-Mass $(\mathrm{kg})$ & 39.02 & 32.94 & 0.001 \\
\hline Muscle Mass $(\mathrm{kg})$ & 37.64 & 32.01 & 0.003 \\
\hline Glucose $(\mathrm{mg} / \mathrm{dl})$ & 82.67 & 81.08 & 0.188 \\
\hline Cholesterol $(\mathrm{mg} / \mathrm{dl})$ & 166.99 & 156.98 & 0.049 \\
\hline Triglycerides $(\mathrm{mg} / \mathrm{dl})$ & 89.87 & 71.24 & $<0.001$ \\
\hline HDL-Cholesterol $(\mathrm{mg} / \mathrm{dl})$ & 51.09 & 55.46 & 0.001 \\
\hline Uric Acid $(\mathrm{mg} / \mathrm{dl})$ & 4.91 & 4.23 & $<0.001$ \\
\hline Insulin $(\mu \mathrm{UI} / \mathrm{ml})$ & 37.01 & 23.47 & $<0.001$ \\
\hline HOMA index & 7.58 & 4.66 & $<0.001$ \\
\hline Visfatin $(\mathrm{ng} / \mathrm{ml})$ & 13507.13 & 16504 & 0.014 \\
\hline
\end{tabular}

BMI: body mass index, HDL: high density lipoprotein, HOMA index: homeostasis model assessment for insulin resistance index, statistical significance was obtained if $\mathrm{p}<0.05$, SD: standard deviation, TST: tricipital skinfold thickness, WHtR: waist per height ratio, Values are expressed as mean. 
$\mathrm{p}=0.001)$, fat-mass $\left(\mathrm{r}_{\mathrm{s}}=0.473, \mathrm{p}=0.001\right)$, free fat-mass $\left(\mathrm{r}_{\mathrm{s}}=0.378, \mathrm{p}=0.001\right)$, muscle mass $\left(\mathrm{r}_{\mathrm{s}}=.344, \mathrm{p}=0.001\right)$, glucose $\left(\mathrm{r}_{\mathrm{s}}=0.281, \mathrm{p}=0.001\right)$, triglycerides $\left(\mathrm{r}_{\mathrm{s}}=0.335, \mathrm{p}=0.001\right)$, HDL-cholesterol $\left(\mathrm{r}_{\mathrm{s}}=-0.166, \mathrm{p}=0.033\right)$. The HOMA index and insulin showed correlations with the same studied parameters, however, in addition to the insulin level, the HOMA index was significantly correlated with uric acid $\left(\mathrm{r}_{\mathrm{s}}=0.181, \mathrm{p}=0.020\right)$. There was no significant correlation between the HOMA index and total cholesterol and visfatin level.

Visfatin level was lower in the case group (13507 vs $16504 \mathrm{ng} / \mathrm{ml}, \mathrm{p}<0.014)$ and was correlated with weight $\left(\mathrm{r}_{\mathrm{s}}=159, \mathrm{p}=0.042\right)$, abdominal circumference $\left(\mathrm{r}_{\mathrm{s}}=-0.200, \mathrm{p}=0.010\right)$, WHtR $\left(\mathrm{r}_{\mathrm{s}}=-\right.$ $0.158, \mathrm{p}=0.040$ ), but not with metabolic profile elements (total cholesterol, triglycerides, fasting glucose, insulin level and HOMA index).

\section{Genetic characteristics}

The allele frequency corresponded to the general data for each polymorphism, and the genotype frequency was in Hardy-Weinberg equilibri- um (for NAMPT rs2302559 $\mathrm{x}^{2}=0.3, \mathrm{p}=0.060$; NAMPT rs4730153 $\left.\mathrm{x}^{2}=0.17, \mathrm{p}=0.069\right)$, however, significant associations were not found between the investigated visfatin SNPs and obesity (Table 2).

There was no significant difference regarding the anthropometric parameters (AC $\mathrm{p}=0.238$, WHtR $\mathrm{p}=0.231$ ), body composition elements (fat mass $\mathrm{p}=0.402$ ), metabolic parameters (HOMA index $\mathrm{p}=0.943)$, and visfatin $(\mathrm{p}=0.147)$ for the $N A M P T$ rs4730153 genotypes (wild-type homozygous genotype AA, heterozygous genotype AG and variant homozygous genotype GG). Considering lipid metabolism, there were statistically significant differences between plasma cholesterol levels according to NAMPT rs4730153 ( $\mathrm{p}=0.030)$ with AG genotype showing the highest level of cholesterol.

NAMPT rs4730153 wild-type homozygous genotype (AA) carriers presented no significant difference between the studied parameters in the investigated groups: anthropometric parameters (BMI $\mathrm{p}=0.803$, AC $\mathrm{p}=0.617$ ), body composition elements (fat mass $\mathrm{p}=0.691$ ), metabolic pa-

Table 2. Genotype distribution of visfatin rs2302559 and rs4730153 SNPs

\begin{tabular}{|c|c|c|c|c|}
\hline NAMPT rs2302559 & $\begin{array}{c}\text { Case (BMI>1 SD) } \\
\text { N=130 }\end{array}$ & $\begin{array}{c}\text { Control } \\
\mathrm{N}=83\end{array}$ & OR $(95 \% C I), p$ value & $\begin{array}{c}\text { Allele frequencies } \\
\%\end{array}$ \\
\hline $\mathrm{C}$ & 112 & 70 & $1.26(0.77-2.06), \mathrm{p}=0.386$ & 66.18 \\
\hline $\mathrm{T}$ & 77 & 38 & - & 33.81 \\
\hline $\mathrm{CC}$ & 49 & 43 & $1.11(0.46-2.72), p=0.825$ & \\
\hline $\mathrm{CT}$ & 63 & 27 & $0.54(0.22-1.35), \mathrm{p}=0.231$ & \\
\hline TT & 14 & 11 & - & \\
\hline $\mathrm{CT}+\mathrm{CC}$ & 112 & 70 & $0.79(0.34-1.85) \mathrm{p}=0.663$ & \\
\hline \multicolumn{5}{|l|}{ NAMPT rs4730153 } \\
\hline $\mathrm{A}$ & 89 & 45 & - & 39.85 \\
\hline $\mathrm{G}$ & 107 & 69 & $1.27(0.79-2.03) \mathrm{p}=0.3423$ & 60.14 \\
\hline AA & 19 & 12 & - & \\
\hline $\mathrm{AG}$ & 70 & 33 & $0.74(0.32-1.71), \mathrm{p}=0.519$ & \\
\hline GG & 37 & 36 & $1.54(0.65-3.62), \mathrm{p}=0.391$ & \\
\hline $\mathrm{GG}+\mathrm{AG}$ & 107 & 69 & $1.02(0.46-2.23), p=0.999$ & \\
\hline
\end{tabular}


rameters (triglycerides $\mathrm{p}=0.922$, HOMA index $\mathrm{p}=0.931)$, and visfatin $(\mathrm{p}=0.170)$. Considering cholesterol levels, there was a statistically significant difference between the two groups in NAMPT rs4730153 AA carriers $(\mathrm{p}=0.036)$.

NAMPT rs4730153 heterozygous genotype (AG) carriers presented no significant difference between the studied parameters in the investigated groups: anthropometric parameters (BMI $\mathrm{p}=0.526$, AC $\mathrm{p}=0.072$ ), body composition elements (fat mass $\mathrm{p}=0.310$ ), metabolic parameters (triglycerides $p=0.056$, HOMA index $p=0.660$ ), and visfatin $(p=0.150)$. However, when considering cholesterol levels, there was a statistically significant difference between the two groups in NAMPT rs4730153 AG carriers $(\mathrm{p}=0.031)$.

NAMPT rs4730153 variant homozygous genotype (GG) carriers presented no significant difference between the studied parameters in the investigated groups: anthropometric parameters (BMI $\mathrm{p}=0.247$, AC $\mathrm{p}=0.188$ ), body composition elements (fat mass $\mathrm{p}=0.188$ ), metabolic parameters (cholesterol $\mathrm{p}=0.452$, triglycerides $\mathrm{p}=0.051$, HOMA index $\mathrm{p}=0.868)$, and visfatin level $(\mathrm{p}=0.171)$.

There was no significant difference regarding the anthropometric parameters (in case of abdominal circumference $\mathrm{p}=0.218$, WHtR $\mathrm{p}=0.269$ ), body composition elements (fat mass $\mathrm{p}=0.186$ ), metabolic parameters (HOMA index $\mathrm{p}=0.703$ ), and visfatin $(\mathrm{p}=0.460)$ for the NAMPT rs 2302559 genotypes (wild-type homozygous genotype TT, heterozygous genotype CT and variant homozygous genotype CC). Considering lipid metabolism, there were statistically significant differences between plasma triglyceride levels according to NAMPT rs2302559 ( $\mathrm{p}=0.045)$, with CT genotype having the highest triglyceride level.

NAMPT rs2302559 wild-type homozygous genotype (TT) carriers presented no significant difference between the studied parameters in the investigated groups: anthropometric parameters
(BMI $\mathrm{p}=0.760, \mathrm{AC} \mathrm{p}=0.400$ ), body composition elements (fat mass $\mathrm{p}=0.995)$, metabolic $\mathrm{pa}$ rameters (HOMA index $\mathrm{p}=0.870$ ), and visfatin $(p=0.160)$. For cholesterol levels, there was a statistically significant difference between the two groups of NAMPT rs2302559 homozygous TT genotype carriers $(\mathrm{p}=0.040)$.

NAMPT rs2302559 heterozygous genotype (CT) carriers presented no significant difference between the studied parameters in the investigated groups: anthropometric parameters (BMI $p=0.237$, AC $p=0.067$ ), body composition elements (fat mass $\mathrm{p}=0.082$ ), metabolic parameters (HOMA index $\mathrm{p}=0.404)$, and visfatin $(\mathrm{p}=0.883)$. Considering the triglycerides levels, there was a statistically significant difference between the two groups of NAMPT rs2302559 heterozygous CT genotype carriers $(\mathrm{p}=0.015)$.

NAMPT rs2302559 variant homozygous genotype (CC) carriers presented no significant difference between the studied parameters in the investigated groups: anthropometric parameters (BMI $\mathrm{p}=0.273$, AC $\mathrm{p}=0.283$ ), body composition elements (fat mass $\mathrm{p}=0.196$ ), metabolic parameters (cholesterol $\mathrm{p}=0.387$, HOMA index $\mathrm{p}=0.791)$, and visfatin $(\mathrm{p}=0.165)$.

\section{Discussions}

Childhood is considered a critical period for the onset of obesity which in turn leads to significant health changes with negative impact on adult's health (27).

The interaction between HOMA-IR and BMI in preadolescence predicts the likelihood of having metabolic syndrome in late adolescence. There are studies that showed a significant positive correlation between insulin levels, HOMA index and weight, BMI, AC and WHtR (28, 29), in concordance with our results. Research demonstrated that increased insulin levels were an independent risk factor for the appearance of cardiovascular conditions, by increasing the risk 
of dyslipidemia (30), among those with hyperinsulinemia, dyslipidemia cases being threefold higher (27). In our study, the HOMA index was significantly correlated with triglycerides, but there was no significant correlation with total cholesterol. Our results were similar to the study performed by Romualdo et al. which revealed that triglyceride levels were significantly higher in participants with insulin resistance, but there was no significant correlation between HOMA index and total cholesterol (31).

Serrano et al. demonstrated that overweight adolescents were 4.5-fold more likely to have changes in the HOMA index, while adolescents with higher fat mass percentage had higher HOMA index values (32). These data support our results, as in our study insulin plasmatic levels and HOMA index were correlated with all body composition components - fat mass, nonfat, and muscle mass. There is still no agreement on the normal values of HOMA-IR for pediatric evaluation, as these values tend to fluctuate greatly, especially at puberty. To more accurately investigate the relationship between HOMA-IR cut-off and associated risk, more prospective studies need to be considered.

Studies which evaluated visfatin plasma levels are highly controversial in both adults and pediatric populations. In our study, visfatin levels were significantly lower in the obese and overweight group compared with the control group, contrary to other studies that showed elevated visfatin levels in obese or overweight patients (33-35), nevertheless these studies included children with obesity and at least three criteria for metabolic syndrome (34) or they were performed on small sample populations (35). However, our results were similar to those of a study conducted on 241 newly diagnosed type 2 diabetes patients and normal glucose tolerant adult subjects which showed that visfatin levels were significantly lower in patients with obesity compared with overweight and normal-weighted subjects (36).
In children, studies have shown a positive correlation between visfatin plasma concentrations and BMI and abdominal circumference (34-36). Similarly, in our study visfatin plasmatic levels were also correlated with BMI, AC, weight, and WHtR. It has been discovered that visfatin levels increase with the number of components of the metabolic syndrome (34). However, our study showed that visfatin plasmatic levels were not correlated with metabolic parameters (cholesterol, triglycerides, glucose or HOMA index), even though in an obese adult population serum visfatin levels were negatively correlated with HDL and positively correlated with serum triglycerides (38).

There are studies suggesting that insulin might be a potential mediator in the visfatin pathway, and there is a stronger link between insulin and visfatin than glucose (39), although other studies revealed controversial results on the expression of visfatin and its relationship with insulin resistance $(35,40)$. The discordance between results could be attributed to the differences in sensitivity and specificity of the available visfatin immunoassay kits. However, in our study, there was no significant correlation between these parameters. Conflicting results considering the relationship between visfatin plasmatic levels and obesity could also be explained by the influence of gene variants involved in the synthesis or action of these hormones. However, literature data about these polymorphisms are controversial and extremely limited.

Considering NAMPT rs2302559, our results showed no significant differences between its genotypes and anthropometric parameters (BMI, AC, WHtR, fat mass), results that are supported by other studies (40). Considering visfatin level, a study which analyzed 243 obese children showed that rs2302559 variant allele carriers had lower fasting serum visfatin and lower fasting plasma glucose as compared with wild-type allele carriers (39), however, in our study, there 
was no significant difference in visfatin levels between NAMPT rs2302559 genotypes.

Regarding the lipid metabolism, in our study, there were statistically significant differences between plasma triglyceride levels according to NAMPT rs2302559 genotypes, with the heterozygous genotype having the highest level of triglycerides. We did not find similar results in the literature.

Considering the NAMPT rs4730153 genotypes, regarding the lipid profile, we found statistically significant differences between plasma cholesterol levels, with carriers of the heterozygote genotype having the highest cholesterol levels, results supported by another study indicating that the same polymorphism was likely to contribute to lipid metabolic malfunction (41). However, these results disagree with other previous studies in which no association between rs4730153 SNP and cholesterol, HDL cholesterol and triglycerides was found $(42,43)$.

In our study, no associations were found between NAMPT rs4730153, anthropometric and body composition parameters, HOMA index and visfatin levels, results supported by other studies (42). However, there was a study on the adult population that showed a significant association of NAMPT rs4730153 variant allele with glucose, insulin and HOMA index (15). In our study, NAMPT rs4730153 variant genotype was not associated with anthropometric parameters, HOMA index or visfatin levels. The reason for the discrepancies among these studies is unclear, but differences in race, the number of the children investigated in different studies, and determination techniques (visfatin immunoassay kits) might contribute to the discordant results.

Finally, some study limitations must be highlighted: obesity is a multifactorial problem (44); genetic, metabolic, social and hormonal factors, lack of physical activity, unhealthy regional eating habits being the most commonly involved ones. We did not evaluate puberty in our chil- dren, a physiological stage known to modify insulin resistance. Also, in the control group, we included children referred to the hospital for minor conditions that might have other characteristics which influence visfatin levels. To the best of our knowledge, our analysis is the first study which analyzes the Eastern European pediatric population. Although this study demonstrated an association between visfatin rs4730153 and rs2302559 SNPs and metabolic factors, the specific pathway by which the studied SNPs influence the cardiometabolic parameters remain to be established by future studies.

In conclusion, NAMPT rs2302559 and rs4730153 polymorphisms do not seem to have a major impact in the development of obesity in children. However, there may be an association with lipid profile, but further studies in order to confirm the association at populational levels are needed. Additional genetic studies in larger study groups are required to perform the profile of these polymorphisms and to confirm the association at populational levels.

\section{Abbreviation}

SNP: single nucleotide polymorphisms

WHO: World Health Organization

IOTF: International Obesity Task Force

AACE: American Association of Clinical Endocrinologist

ACE: American College of Endocrinology

ABCD: Adiposity-based chronic disease

RBP4: retinol binding protein 4

IR: insulin resistance

HOMA - IR: homeostasis model assessment for insulin resistance index

NAMPT: nicotinamide phosphoribosyltransferase

RNA: Ribonucleic acid

BMI SD: body mass index standard deviation score

AC: abdominal circumference 
SD: standard deviation score

TST: Tricipital skinfold thickness

WHtR: waist to height

HDL: high-density lipoprotein

EDTA: Ethylenediaminetetraacetic acid

CVs: coefficients of variations

OD: odds ratio

CI: confidence interval

\section{Acknowledgements}

This study was partially supported by an internal research grant from the University of Medicine and Pharmacy of Tirgu Mures, Romania (No. $17802 / 1 / 22.12 .2015)$

\section{Author Contributions}

SLV (Conceptualization, Investigation, Writing-original draft presentation); IMP (Conceptualization, Resources, Writing-review and editing);

$\mathrm{AB}$ (Investigation)

RMP (Formal analysis, Writing-review and editing);

CB (Genotyping, Conceptualization, Resources, critical review of the manuscript and editing); VM (DNA isolation, Genotyping, Resources); $\mathrm{AH}$ (Visfatin quantification, Resources); CD (Sample and data collection, Writing-review and editing);

COM (Conceptualization, Resources, Writing-review and editing).

\section{Conflict of Interest Statement}

The authors have no conflicts of interest to declare.

\section{References}

1. Cinteza EE, Cinteza M. Biomarkers in Obesity. Rev Romana Med Lab. 2018;26(3):353-8. DOI: 10.2478/ rrlm-2018-0027

2. Duicu C, Mărginean CO, Voidăzan S, Tripon F, Bănes- cu C. FTO rs 9939609 SNP Is Associated With Adiponectin and Leptin Levels and the Risk of Obesity in a Cohort of Romanian Children Population. Medicine (Baltimore). 2016 May;95(20):e3709. DOI: 10.1097/ MD.0000000000003709

3. WHO | Commission on Ending Childhood Obesity. WHO [cited 2019 August 28];Available from: http:// www.who.int/end-childhood-obesity/en/

4. Cole TJ, Lobstein T. Extended international (IOTF) body mass index cut-offs for thinness, overweight and obesity. Pediatr Obes 2012;7:284-94 DOI: 10.1111/j.2047-6310.2012.00064.x

5. Pascanu I, Pop R, Barbu CG, Dumitrescu CP, Gherlan I, Marginean $\mathrm{O}$, et al. development of synthetic growth charts for romanian population. Acta Endocrinol (Buchar). 2016 Jul-Sep;12(3):309-18. DOI: 10.4183/ aeb.2016.309

6. Popa S, Moţa M, Popa A, Moţa E, Serafinceanu C, Guja C, et al. Prevalence of overweight/obesity, abdominal obesity and metabolic syndrome and atypical cardiometabolic phenotypes in the adult Romanian population: PREDATORR study. J Endocrinol Invest 2016;39(9): 1045-53. DOI: 10.1007/s40618-016-04704

7. Chirita-Emandi A, Barbu CG, Cinteza EE, Chesaru BI, Gafencu M, Mocanu V, et al. Overweight and Underweight Prevalence Trends in Children from Romania - Pooled Analysis of Cross-Sectional Studies between 2006 and 2015. Obes Facts. 2016;9(3):206-20. DOI: 10.1159/000444173

8. Mechanick JI, Hurley DL, Garvey WT. Adiposity-based chronic disease as a new diagnostic term: The American Association of Clinical Endocrinologists and American College of Endocrinology Position statement. Endocr Pract. 2017 Mar;23(3):372-78. DOI: 10.4158/EP161688.PS

9. Leal Vde O, Mafra D. Adipokines in obesity. Clin Chim Acta. 2013 Apr;419:87-94. DOI: 10.1016/j. cca.2013.02.003

10. Bahíllo-Curieses MP, Hermoso-López F, Martínez-Sopena MJ, Cobreros-García P, García-Saseta P, Tríguez-García M, et al. Prevalence of insulin resistance and impaired glucose tolerance in a sample of obese Spanish children and adolescents. Endocrine. 2012 Apr;41(2):289-95. DOI: 10.1007/s12020-0119540-8

11. Damiani D, Kuba VM, Cominato L, Damiani D, 
Dichtchekenian V, Menezes HC. Metabolic syndrome in children and adolescents: doubts about terminology but not about cardiometabolic risks. Arq Bras Endocrinol Metabol. 2011 Nov;55(8):576-82. DOI: 10.1590/ S0004-27302011000800011

12. Stastny J, Bienertova-Vasku J, Vasku A. Visfatin and its role in obesity development. Diabetes Metab Syndr. 2012 Apr-Jun;6(2):120-4. DOI: 10.1016/j. dsx.2012.08.011

13. Garten A, Petzold S, Barnikol-Oettler A, Körner A, Thasler WE, Kratzsch J, et al. Nicotinamide phosphoribosyltransferase (NAMPT/PBEF/visfatin) is constitutively released from human hepatocytes. Biochem Biophys Res Commun. 2010 Jan;391(1):376-81. DOI: 10.1016/j.bbrc.2009.11.066

14. Costford SR, Bajpeyi S, Pasarica M, Albarado DC, Thomas SC, Xie H, et al. Skeletal muscle NAMPT is induced by exercise in humans. Am J Physiol Endocrinol Metab. 2010 Jan;298(1):E117-26. DOI: 10.1152/ ajpendo.00318.2009

15. Martínez Larrad MT, Corbatón Anchuelo A, Fernández Pérez C, Pérez Barba M, Lazcano Redondo Y, Serrano Ríos M, et al. Obesity and Cardiovascular Risk: Variations in Visfatin Gene Can Modify the Obesity Associated Cardiovascular Risk. Results from the Segovia Population Based-Study. Spain. PloS One. 2016 May;11(5):e0153976. DOI: 10.1371/journal. pone. 0153976

16. Alkhzouz C, Miclea D, Farcas M, Bucerzan S, Cabau G, Popp RA. Is there a correlation between GAD2 gene -243 A $>$ G polymorphism and obesity? Rev Romana Med Lab. 2019;27(4):413-20. DOI: 10.2478/rrlm2019-0033

17. Pigeyre M, Yazdi FT, Kaur Y, Meyre D. Recent progress in genetics, epigenetics and metagenomics unveils the pathophysiology of human obesity. Clin Science. 2016 Jun;130(12):943-86. DOI: 10.1042/CS20160136

18. World Health Organization. Growth reference 5-19 years. 2007. WHO [cited 2019 August 28]; Available from: http://www.who.int/growthref/who2007_bmi for_age/en/

19. Prader A, Largo RH, Molinari L, Issler C. Physical growth of Swiss children from birth to 20 years of age. First Zurich longitudinal study of growth and development. Helv Paediatr Acta Suppl. 1989 Jun;52:1-125.

20. National Health and Nutrition Examination Survey. Anthropometry Procedures Manual. 2017. Available from: https://wwwn.cdc.gov/nchs/data/nhanes/2017-2018/ manuals/2017_Anthropometry_Procedures_Manual. pdf

21. WHO. Waist circumference and waist-hip ratio. WHO [cited 2019 August 28]; Available from: http://www. who.int/nutrition/publications/obesity/WHO_report_ waistcircumference_and_waisthip_ratio/en/

22. Martin-Calvo N, Moreno-Galarraga L, Martinez-Gonzalez MA. Association between Body Mass Index, Waist-to-Height Ratio and Adiposity in Children: A Systematic Review and Meta-Analysis. Nutrients. 2016;8(8), pii: E512. DOI: 10.3390/nu8080512

23. Tanita Corporation of America Inc. „TANITA - Total Body Composition Analyzer SC-331S - Instruction Manual. 2016.

24. Mihai G, Gasparik AI, Pascanu IM, Cevei M, Hutanu A, Pop RM. The influence of Visfatin, RBP-4 and insulin resistance on bone mineral density in women with treated primary osteoporosis. Aging Clin Exp Res. 2019 Jun;31(6):889-95. DOI: 10.1007/s40520-019-01206-6

25. Matthews DR, Hosker JP, Rudenski AS, Naylor BA, Treacher DF, Turner RC. Homeostasis model assessment: insulin resistance and beta-cell function from fasting plasma glucose and insulin concentrations in man. Diabetologia. 1985 Jul;28(7):412-9. DOI: 10.1007/BF00280883

26. Andrade MI, Oliveira JS, Leal VS, Lima NM, Costa $\mathrm{EC}$, Aquino NB, et al. Identification of cutoff points for Homeostatic Model Assessment for Insulin Resistance index in adolescents: systematic review. Rev Paul Pediatr. 2016 Jun;34(2):234-42. DOI: 10.1016/j. rppede.2016.01.004

27. Ricco RC, Ricco RG, Almeida CAN, Ramos APP. Comparative study of risk factors among children and adolescents with an anthropometric diagnosis of overweight or obesity. Rev Paul Pediatr. 2010;28(4):320-5. DOI: 10.1590/S0103-05822010000400006

28. Castro AV, Kolka CM, Kim SP, Bergman RN. Obesity, insulin resistance and comorbidities? Mechanisms of association. Arq Bras Endocrinol Metabol. 2014 Aug;58(6):600-9. DOI: 10.1590/0004-2730000003223

29. Kostovski M, Simeonovski V, Mironska K, Tasic V, Gucev Z. Metabolic Profiles in Obese Children and Adolescents with Insulin Resistance. Open Access Maced J Med Sci. 2018 Mar;6(3):511-8 DOI: 10.3889/oamjms.2018.097

30. Pinhas-Hamiel O, Lerner-Geva L, Copperman NM, Ja- 
cobson MS. Lipid and insulin levels in obese children: changes with age and puberty. Obes Silver Spring Md. 2007 Nov;15(11):2825-31. DOI: 10.1038/oby.2007.335

31. Romualdo MC, Nóbrega FJ, Escrivão MA. Insulin resistance in obese children and adolescents. J Pediatr (Rio J). 2014 Nov-Dec;90(6):600-7. DOI: 10.1016/j. jped.2014.03.005

32. Serrano HM, Carvalho GQ, Pereira PF, Peluzio Mdo C, Franceschini Sdo C, Priore SE. Body composition, biochemical and clinical changes of adolescents with excessive adiposity. Arq Bras Cardiol. 2010 Oct;95(4):46472. DOI: $10.1590 / \mathrm{S} 0066-782 X 2010005000109$

33. Chang YH, Chang DM, Lin KC, Shin SJ, Lee YJ. Visfatin in overweight/obesity, type 2 diabetes mellitus, insulin resistance, metabolic syndrome and cardiovascular diseases: a meta-analysis and systemic review. Diabetes Metab Res Rev. 2011 Sept;27(6):515-27. DOI: 10.1002/dmrr.1201

34. Kolsgaard ML, Wangensteen T, Brunborg C, Joner G, Holven KB, Halvorsen B, et al. Elevated visfatin levels in overweight and obese children and adolescents with metabolic syndrome. Scand J Clin Lab Invest. 2009;69:858-64. DOI: 10.3109/00365510903348677

35. Salama HM, Galal A, Motawie AA, Kamel AF, Ibrahim DM, Aly AA, et al. Adipokines Vaspin and Visfatin in Obese Children. Open Access Maced J Med Sci. 2015 Dec;3(4):563-6. DOI: 10.3889/oamjms.2015.123

36. Jian WX, Luo TH, Gu YY, Zhang HL, Zheng S, Dai $\mathrm{M}$, et al. The visfatin gene is associated with glucose and lipid metabolism in a Chinese population. Diabet Med J Br Diabet Assoc. 2006 Sept;23(9):967-73. DOI: 10.1111/j.1464-5491.2006.01909.x

37. Li RZ, Ma Xn, Hu XF, Kang SX, Chen SK, Cianflone $\mathrm{K}$, et al. Elevated visfatin levels in obese children are related to proinflammatory factors. J Pediatr Endocrinol Metab. 2013;26(1-2):111-8.

38. Filippatos TD, Derdemezis CS, Kiortsis DN, Tselepis
$A D$, Elisaf MS. Increased plasma levels of visfatin/ pre-B cell colony-enhancing factor in obese and overweight patients with metabolic syndrome. J Endocrinol Invest. 2007 Apr;30(4):323-6. DOI: 10.1007/ BF03346300

39. Ooi SQ, Chan RM, Poh LK, Loke KY, Heng CK, $\mathrm{Chan} \mathrm{YH}$, et al. Visfatin and its genetic variants are associated with obesity-related morbidities and cardiometabolic risk in severely obese children. Pediatr Obes. 2014 Apr;9(2):81-91. DOI: 10.1111/j.20476310.2013.00149.x

40. Stastny J, Bienertova-Vasku J, Tomandl J, Tomandlova $\mathrm{M}$, Zlamal F, Forejt M, et al. Association of genetic variability in selected regions in visfatin (NAMPT) gene with anthropometric parameters and dietary composition in obese and non-obese Central-European population. Diabetes Metab Syndr. 2013 Jul-Sep;7(3):166-71. DOI: 10.1016/j.dsx.2013.06.001

41. Lai A, Chen W, Helm K. Effects of visfatin gene polymorphism RS4730153 on exercise-induced weight loss of obese children and adolescents of Han Chinese. Int J Biol Sci. 2013;9(1):16-21 DOI: 10.7150/ijbs.4918

42. Körner A, Böttcher Y, Enigk B, Kiess W, Stumvoll M, Kovacs P. Effects of genetic variation in the visfatin gene (PBEF1) on obesity, glucose metabolism, and blood pressure in children. Metabolism. 2007 Jun;56(6):772-7. DOI: 10.1016/j.metabol.2007.01.009

43. Ferrari G, Lima Rodrigues J, Fernandes I, Bueno Jr C. Association between rs4730153 Gene SNP and fasting glucose, triglyceride, HDL and body mass index levels in overweight brazilian adults. Int J Cardiovasc Sci. 2016;29(6):471-76. DOI: 10.5935/23594802.20160067

44. Duicu C. Genetic testing in pediatrics - a narrative essay of challenges and possibilities in Romania. Rev Romana Med Lab. 2019;27(4):355-9. DOI: 10.2478/ rrlm-2019-0041 